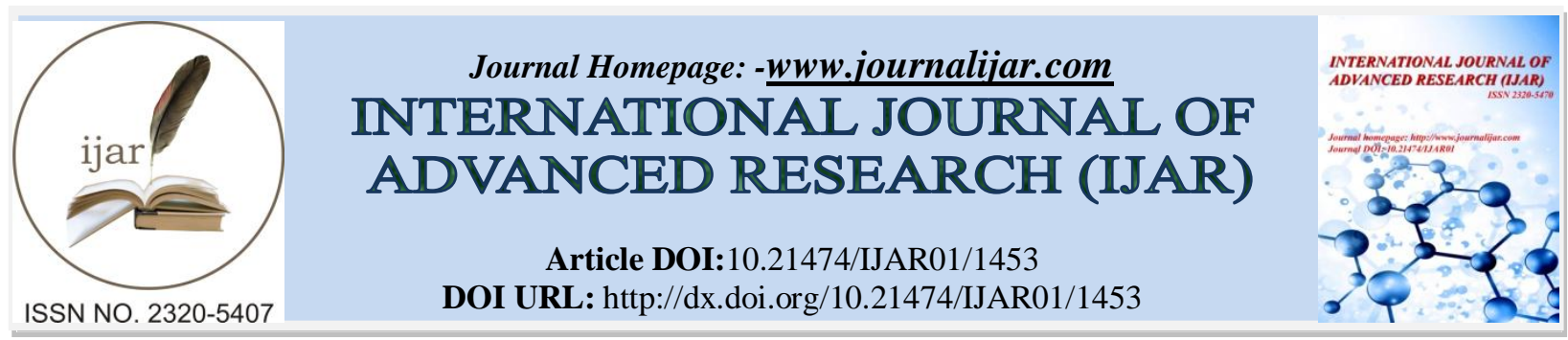

RESEARCH ARTICLE

\title{
SEX HORMONE-BINDING GLOBULIN IN OBESE TYPE 2 DIABETES MELLITUS.
}

Rehab A Mohamed ${ }^{1}$. Nagwa A Mohamed ${ }^{2}$, Hend M Maghraby ${ }^{11}$ and Eman M Abd EL Salam ${ }^{1}$.

1. Internal Medicine Departments, Al Azhar University, Cairo, Egypt.

2. Department of Clinical and Chemical Pathology, National Research Center, Cairo, Egypt.

\section{Manuscript Info}

Manuscript History

Received: 12 July 2016

Final Accepted: 19 August 2016

Published: September 2016

Key words:-

sex hormone binding globulin, Insulin resistance, type 2 diabetes mellitus, Obesity.

\section{Abstract}

Background:- Diabetes Mellitus is a public health problem of pandemic proportions.Most patients with type 2 diabetes are obese with variable degrees of gender-related adiposity and fat tissue distribution. Obesity is one of risk factors for type 2 diabetes because of its association with insulin resistance and glycemic control impairment. A great concern in respect to the role of Sex hormonebinding globulin (SHBG) and adipose tissue hormones in the development of insulin resistance, hyperlipidemia and type 2diabetes was paid. Previous studies suggested that serum sex hormone-binding globulin (SHBG) has a role in glucose homeostasis in both men and women and low levels are associated with development of diabetes,insulin resistance and hyperinsulinemia. Aim of the work: to determine the relationship between serum sex hormone-binding globulin,obesity, and metabolic parameters in type 2 diabetes in both sex.

Methods: forty obese type 2 diabetic patients were selected and compared with ten obese non-diabetics as control. After complete examination; insulin level, fasting blood glucose, postprandial glucose, glycated hemoglobin ( $\mathrm{HbA} 1 \mathrm{c})$, cholesterol, triglycerides and serum levels of sex hormone binding globulin were measured. Results: the mean SHBG concentration was lower in diabetic group $(18.95 \pm 3.4 \mathrm{nmol} / \mathrm{L})$ than non-diabetic group $(35.17 \pm 8 \mathrm{nmol} / \mathrm{L})$. There were significant inverse associations between SHBG, age, disease duration, body mass index, fasting blood sugar, postprandial glucose. On the other hand, there were no significant correlations between SHBG and waist circumference, fasting insulin, HOMA-IR, cholesterol and triglyceride levels.

Conclusion: low SHBG is associated with hyperglycemia in both males and females, independently of insulinemia.

Copy Right, IJAR, 2016,. All rights reserved.

\section{Introduction:-}

Diabetes Mellitus is a metabolic disease characterized by hyperglycemia due to defects in insulin secretion, insulin action, or both. The chronic hyperglycemia of diabetes is associated with long-term dysfunction, damage and failure of different organs, especially the eyes, kidneys, nerves, heart, and blood vessels $\mathbf{1}$. World Health Organization (WHO) in 2014 reported that the global prevalence of diabetes was close to $10 \%$ among adults aged 18 years and 
above $^{2}$. The WHO survey showed the prevalence of known diabetes to be 6.0\% in Egypt. By 2030, Egypt will have about 8.6 million adults with diabetes and will be the tenth largest population of diabetics in the world. Diabetes Mellitus is the eleventh most important cause of premature mortality in Egypt ${ }^{3}$. Early diagnosis of diabetes mellitus is limited to serum glucose and glycated hemoglobin (HbA1c). Serum glucose testing is affected byshort-term lifestyle changes, lower reproducibility and sample processing. The use of HbAlc for screening and diagnosing diabetes mellitus also has limitations, as it is only able to reflect glycemic control in retrospect and is affected by hemolytic anemia ${ }^{4}$. Many factors contribute to physiologic changes responsible for type 2 diabetes (T2DM). Many conditions of hyperandrogenism in women, such as in polycystic ovarian syndrome (PCO) and hypoandrogenism in men is linked to insulin resistance, suggesting that alterations in normal sex steroids may play a role in the pathogenesis of diabetes.

SHBG is identified as a contributing factor in the pathophysiology of T2DM. Many studies demonstrating a relationship between decreased serum levels of SHBG and T2DM, also genetic studies revealed that transmission of specific polymorphisms in the SHBG gene influence the risk of $\mathrm{T}_{2} \mathrm{DM}^{5}$. At the molecular level, the multiple interactions between SHBG and its receptors in various target tissues suggest physiologic roles for SHBG that are more complex than the simple transport of sex hormones in serum ${ }^{6}$.SHBG may contribute to the impairment of glucose metabolism through modulation of sex hormones bioavailability and direct activation of specific receptor for SHBG. Rosner et al, ${ }^{7}$ found that plasma membranes of different types of cells are capable of binding specifically and with high affinity to SHBG. Taken together, these data support the role of SHBG in the pathophysiology of insulin resistance and T2DM. SHBG is a stable protein with a relatively constant diurnal pattern, and its concentrations tend to be more reliably measured than those of sex steroids ${ }^{8}$.

Previous studies identified the effect of different hormonal, genetic and lifestyle-related factors on circulating SHBG levels. Classically, the estrogen/ androgen balance was the main factor in the regulation of SHBG synthesis. At present, SHBG regulation is considered as multifactorial and other non-steroidal factors have an important role in the circulating levels of this binding protein. In obese persons, SHBG levels were found to be lower than in normal weight people and weight loss through calorie restriction is found to increase serum SHBG as well as insulin sensitivity supporting that body composition is involved in the determination of SHBG levels?

\section{Methods:-}

Participants in this case control study were recruited from inpatient and outpatient clinic of Internal medicine department, AL-Zahraa University Hospital. Study participant $(n=50)$ were classified into, Group I: forty obese subjects with T2DM were defined to have diabetes according to American Diabetes Association criteria fasting plasma glucose levels $\geq 126 \mathrm{mg} / \mathrm{dl}$, 2-h postprandial glucose levels $\geq 200 \mathrm{mg} / \mathrm{dl}{ }^{\mathbf{1 0}}$ or they on current treatment by oral glucose lowering agents. They further classified into male group: sixteen obese diabetic patients and Female group: twenty-four obese diabetic females.

Group II: Ten obese subjects without history of hormonal disturbance or diabetes mellitus who's fasting blood glucose (FBG ) was less than $126 \mathrm{mg} / \mathrm{dl}$ and were matched for age and body mass index (BMI) were included as control group.

Females on contraceptive pills or hormonal replacement therapy, patients with chronic liver or renal diseases and those on medication such as insulin, steroids, anticonvulsants, beta-blockers, thiazides were excluded.

After informed consent all patient were subjected to:

Full medical history and physical Examination:- includingage, disease duration, and drug treatment. Waist circumferences were measured and BMI was calculated by dividing weight (in $\mathrm{kg}$ ) by the square of height (in meters) $\left(\mathrm{Kg} / \mathrm{m}^{2}\right)$. Obesity was defined as BMI $>30$ and waist circumferences $>102 \mathrm{~cm}$ for men and $>88 \mathrm{~cm}$ in women. Clinical investigations include (pelvi-abdominal ultrasonography).

\section{Sampling:-}

Five $\mathrm{ml}$ of fasting (12-14 hours) venous blood samples were taken from each subject participating in the study and divided into 2 parts: The $1^{\text {st }}$ part $(2 \mathrm{ml})$ was added to a tube containing EDTA for determination of complete blood picture on Coulter Counter T890 (Coulter Counter, Harpenden, UK) and HbA1c cation exchange resin. The $2^{\text {nd }}$ part $(1.4 \mathrm{ml})$ was left to clot and the serum was separated by centrifugation for 15 minutes at $1000 \mathrm{xg}$ and fasting blood glucose was determined immediately on Hitachi auto analyzer (Hitachi 912) by glucose oxidase method. 
The rest of the serum was storedat $-20^{\circ} \mathrm{C}$ for determination of the followings: kidney function tests, liver function tests, lipid profile, insulin and SHBG. $2 \mathrm{ml}$ was taken on fluoride for postprandial (PP) blood glucose 2 hours after meal was determined on Hitachi auto analyzer 912.

\section{Laboratory investigations:}

Kidney function and liver function tests, blood glucose, total cholesterol and triglycerides, HbAlc, were performed on Hitachi auto analyzer 912 (Roche Diagnostics GmbH, D-68298 Mannheim, USA) by colorimetric techniques. Complete urine analysis was done. Fasting serum insulin was determined using RIA ${ }^{11}$. Measurement of serum SHBG was performed by electro chemi luminescence immunoassay on Elecsys 2010 auto analyzer (Roche Diagnostics) ${ }^{\mathbf{1 2}}$.

The insulin resistance was calculated using the HOMA-IR method $($ HOMA-IR $=[$ insulin $\mu$ iu $\times$ glucose mg/dl $] / 405)$ and is considered elevated at HOMA-IR $>3^{\mathbf{1 3}}$.

\section{Statistical Analysis:-}

Results were collected, tabulated, and statistically analyzed by personal computer and statistical package SPSS version 10 (Chicago, USA).Two types of statistics were performed: Descriptive statistics - for example, mean $(X)$ and SD - and analytic statistics. Quantitative variables were compared using unpaired t-test. Spearman correlation coefficient ( $r$ ) is a test used to measure the association between two quantitative variables. The $P$ value of less than 0.05 was considered as statistically significant.

\section{Results:-}

Forty obese T2DM patients were included in this study, their mean ages were $52.35 \pm 6.16$, and their disease duration was $8.97 \pm 4.40$ years. BMI was $32.92 \pm 3.88$ and waist Circumference was $98.3 \pm 7.06$.

There were no statistical significant differences between patients and controls regarding age or anthropometric measures ( $p>0.05)$. Main clinical characteristics of the population studied are shown in (Table 1).

There were statistical significant increase in fasting blood glucose (FBG), fasting insulin, and two hours postprandial glucose (PP), HOMA-IR, HbAlc in diabetic patients compared to control group ( $<$ < 0.001) (Table 1).

Comparing other variables, the mean serum total cholesterol and the mean LDL cholesterol in the diabetic obese patients were $193.1 \mathrm{mg} / \mathrm{dl}$ and $137.67 \mathrm{mg} / \mathrm{dl}$ respectively which were significantly higher than the control group with p-values of $<0.001$. The mean serum SHBG of the control group was $35.17 \mathrm{nmol} / \mathrm{L}$ whereas that of the diabetic group was $18.35 \mathrm{nmol} / \mathrm{L}$. The difference between the two groups was extremely significant with a p-value of $<0.001$. There was significant decrease in HDL cholesterol in diabetic patients compared to control group ( $\mathrm{p}<$ 0.001) (Table 1).

The serum concentration of SHBG was significantly negatively correlated with age, disease duration, BMI, FBS, PP; while there were no significant correlations between SHBG and waist circumference, fasting insulin, HbAlc, HOMA-IR, cholesterol and triglyceride levels (Table 2).

No gender differences were observed with regard to clinical or laboratory parameters (Table 3). By comparing male and female patients there was only significant negative correlation between SHBG and BMI in female diabetic patients and FBS in male diabetic patients ( $p<0.001)$ (Table 4).

Table 1:-Comparison of variables between diabetic patients and control group

\begin{tabular}{|l|l|c|c|}
\hline Variable & T2DM group $(\mathbf{n = 4 0})$ & Non-diabetic Controlgroup $(\mathbf{n}=\mathbf{1 0})$ & P value \\
\hline Age $($ year) & $52.35 \pm 6.163$ & $51.8 \pm 7.480$ & 0.833 \\
\hline FBS $(\mathrm{mg} / \mathrm{dl})$ & $152.55 \pm 40.449$ & $93 \pm 4.082$ & $0.000^{* *}$ \\
\hline PP $(\mathrm{mg} / \mathrm{dl})$ & $219.45 \pm 53.482$ & $117.3 \pm 10.122$ & $0.000^{* *}$ \\
\hline HbAlc $(\%)$ & $7.15 \pm 1.159$ & $5.71 \pm 0.288$ & $0.000^{* *}$ \\
\hline Fasting insulin $(\mu \mathrm{iu})$ & $34.3 \pm 7.800$ & $17.98 \pm 2.439$ & $0.000^{* *}$ \\
\hline Total cholesterol $(\mathrm{TC})(\mathrm{mg} / \mathrm{dl})$ & $193.1 \pm 52.781$ & $120.3 \pm 8.782$ & $0.000^{* *}$ \\
\hline Triglycerides $(\mathrm{mg} / \mathrm{dl})$ & $195.075 \pm 56.448$ & $132.5 \pm 8.972$ & $0.000^{* *}$ \\
\hline
\end{tabular}




\begin{tabular}{|l|l|c|c|}
\hline HDL-cholesterol (mg/dl) & $36.925 \pm 9.678$ & $47.3 \pm 4.373$ & $0.000^{* *}$ \\
\hline LDL $(\mathrm{mg} / \mathrm{dl})$ & $137.675 \pm 18.570$ & $60.9 \pm 9.562$ & $0.000^{* *}$ \\
\hline SHBG $(\mathrm{nmol} / \mathrm{L})$ & $18.95 \pm 3.423$ & $35.17 \pm 8.229$ & $0.000^{* *}$ \\
\hline HOMA-IR & $154.05 \pm 40.408$ & $73.9 \pm 10.12$ & $0.000^{* *}$ \\
\hline BMI $(\mathrm{kg} / \mathrm{m} 2)$ & $32.92 \pm 3.88$ & $32.33 \pm 2.30$ & 0.645 \\
\hline Waist Circumference $(\mathrm{cm})$ & $98.3 \pm 7.06$ & $97.7 \pm 9.3$ & 0.834 \\
\hline
\end{tabular}

FBS = fasting blood sugar, HOMA = homeostatic model assessment, IR = insulin resistance, HDL=high density lipoprotein, $\mathrm{LDL}=$ low density lipoprotein, $\mathrm{SHBG}=$ sex hormone-binding globulin, $\mathrm{BMI}=$ body mass Index $\mathrm{P}$ value $<0.05^{*}$, $\mathrm{p}$ value $<0.001^{* *}$, $\mathrm{p}$ value $>0.05$

Table 2:-Correlation of SHBG with clinical and laboratory parameters in diabetic group

\begin{tabular}{|l|c|c|}
\hline \multicolumn{1}{|c|}{ Variable } & r value & p value \\
\hline Age & -0.150 & $0.025^{*}$ \\
\hline Disease duration & 0.197 & $0.038^{*}$ \\
\hline BMI & -0.364 & $0.021^{*}$ \\
\hline Waist circumference & -0.120 & 0.462 \\
\hline FBS & -0.645 & $0.000^{* *}$ \\
\hline PP & -0.403 & $0.000^{* *}$ \\
\hline HbAlc & 0.129 & 0.428 \\
\hline Fasting insulin & -0.114 & 0.485 \\
\hline HOMA-IR & 0.114 & 0.485 \\
\hline Total cholesterol & 0.026 & 0.873 \\
\hline Triglyceride & 0.162 & 0.318 \\
\hline LDL & 0.097 & 0.551 \\
\hline
\end{tabular}

Table 3:- Comparison of variables between male and female diabetic patients

\begin{tabular}{|l|c|c|l|}
\hline \multicolumn{1}{|c|}{ Variable } & Female $(\mathbf{n = 2 4})$ & Male $(\mathbf{n}=\mathbf{1 6})$ & P- value \\
\hline & Mean \pm SD & Mean \pm SD & \\
\hline Age $($ year $)$ & $49.58 \pm 7.31$ & $51.50 \pm 3.79$ & 0.342 \\
\hline SHBG $(\mathrm{nmol})$ & $19.85 \pm 3.34$ & $17.59 \pm 3.18$ & 0.039 \\
\hline Fasting insulin $(\mu \mathrm{iu})$ & $34.03 \pm 8.45$ & $34.71 \pm 6.96$ & 0.789 \\
\hline BMI $(\mathrm{Kg} / \mathrm{m} 2)$ & $31.88 \pm 4.42$ & $31.99 \pm 3.02$ & 0.926 \\
\hline HOMO- IR & $154.17 \pm 40.62$ & $153.88 \pm 41.41$ & 0.983 \\
\hline Waist circumference $(\mathrm{cm})$ & $97.13 \pm 8.13$ & $96.31 \pm 6.62$ & 0.741 \\
\hline Total cholesterol $(\mathrm{TC})(\mathrm{mg} / \mathrm{dl})$ & $186.29 \pm 44.67$ & $203.31 \pm 63.23$ & 0.324 \\
& & & \\
\hline Triglycerides $(\mathrm{mg} / \mathrm{dl})$ & $201.79 \pm 49.27$ & $185.00 \pm 66.20$ & 0.363 \\
\hline
\end{tabular}

Table 4:-Correlation of SHBG with variables in male and female diabetic patients

\begin{tabular}{|l|c|c|c|c|}
\hline \multirow{2}{*}{ Variable } & \multicolumn{2}{c|}{$\begin{array}{c}\text { Male patients } \\
\text { n=16 }\end{array}$} & \multicolumn{2}{c|}{$\begin{array}{c}\text { Female patients } \\
\text { n=24 }\end{array}$} \\
\cline { 2 - 5 } & r value & P value & r value & P value \\
\hline Age & -0.259 & 0.333 & -0.062 & 0.773 \\
\hline FBS & -0.702 & $0.002^{*}$ & -0.514 & 0.01 \\
\hline Insulin & 0.084 & 0.757 & 0.118 & 0.582 \\
\hline HOMA-IR & 0.303 & 0.254 & 0.094 & 0.661 \\
\hline Waist circumference & -0.234 & 0.272 & 0.027 & 0.921 \\
\hline BMI & 0.155 & 0.568 & -0.613 & $0.001^{* *}$ \\
\hline HbA1c & -0.103 & 0.704 & 0.029 & 0.891 \\
\hline
\end{tabular}

\section{Discussion:-}

Sex hormone-binding globulin is a glycosylated homodimeric plasma transport protein and is mainly produced in the liver. SHBG is shown to be inversely related to insulin resistance and low SHBG concentrations contributes to the pathophysiology T2DM. Several potential mechanisms may account for this association ${ }^{14}$. Many studies 
implicated insulin as a direct or indirect suppressor of hepatic SHBG production by decreasing total protein secretion in human hepatic cells, while others suggest that excess carbohydrate consumption and levels of fasting glycemia, rather than hyperinsulinemia are the actual determinants of liver SHBG production through down regulation of hepatocyte nuclear factor $4 \alpha(\mathrm{HNF}-4 \alpha)$ activity. Regardless of the mechanism, SHBG is commonly associated with insulin resistance and development of T2DM in overweight populations ${ }^{15}$. The involvement of circulating SHBG in biological functions is attributed to its regulation of bioavailable sex hormone concentrations. Both testosterone and estradiol regulate SHBG levels and have been associated with the development of type 2 diabetes ${ }^{\mathbf{1 6}}$. Low testosterone leads to a decrease in muscle mass and an increase in circulating free fatty acids. Free fatty acids mediate the development of insulin resistance and overt type $2 \mathrm{DM}^{15}$, therefore, sex hormones may partially explain the association between SHBG and diabetes. However, Chen et al., ${ }^{8}$ concluded that a large portion of SHBG's influence on clinical diabetes is independent of free or total sex hormone concentrations, suggesting that the association between SHBG and diabetes could not be completely attributed to confounding or mediation by sex hormone concentrations.

Because SHBG concentrations differ between men and women, the association between variables and diabetes may differ by sex. We aimed to investigate the relationship between SHBG concentration and type 2 diabetes separately in men and women. No gender differences were observed with regard to clinical and laboratory parameters

In this study, diabetic patients had significantly lower levels of SHBG than non-diabetics.Analysisof 23 crosssectional studies found that significantly lower SHBG levels in T2DM in both male and femalecompared with nondiabetic ${ }^{17}$.

In agreement with Peter et al., ${ }^{18}$ we found a strong negative correlation between SHBG, fasting and postprandial glucose levels.Colangelo et al., ${ }^{19}$ observed a significant inverse association between SHBG levels and fasting glucose levels in type 2 diabetes of various ethnicities.

Simó et al., ${ }^{20}$ demonstrated that excessive intake of monosaccharaides leads to lower human SHBG production by reducing hepatocyte nuclear factor 4- $\alpha$ (HNF4- $\alpha$ ) a key transcription factor that regulates $S H B G$ expression in the liver. Functional HNF4 $\alpha$-binding sites are found in over 140 genes, including those involved in the metabolism of glucose, lipids, and amino acids.

Chaoyang et al., ${ }^{21}$ used the homeostasis model assessment of insulin resistance (HOMA-IR) to assess the association between SHBG concentration and insulin resistance and they found that low SHBG is associated with an elevated HOMA-IR in both men and women. In this study, the association between SHBG, insulin and HOMA-IR was insignificant. Fasting insulin did not reach statistical significance at the 0.05 level. This is in agreement with Sutton-Tyrrell et al., ${ }^{22}$ who found that low circulating SHBG was not associated with elevated insulin levels. Selva and colleagues, ${ }^{23}$ demonstrated that hepatic $S H B G$ transcription is mainly responsive to levels of monosaccharides rather than insulin. These finding argue against the hypothesis that hyperinsulinemia is responsible for decreased SHBG levels and support that glucose per se has a major role in the control of SHBG levels.

This finding differs from that of Osuana et al., ${ }^{24}$ who found negative correlation between fasting insulin level and SHBG levels in men.

In this study, SHBG is negatively correlated with age of the diabetic patients, this agrees with the studies of Onatet al., ${ }^{25}$ who found age-related decline in SHBG. There was significant negative correlation between SHBG and body mass index(BMI) and not waist circumference. Body mass index is a major determinant of sex hormone-binding globulin and low serum SHBG levels in overweight individuals are a biomarker for the metabolic syndrome and are predictive of type 2 diabetes ${ }^{26}$. Obesity is considered a state of chronic low-grade inflammation,an inverse correlation was found between SHBG and C-reactive protein levels ${ }^{27}$. In obese subjects, tumor necrosis factor- $\alpha$ (TNF- $\alpha$ ) is mainly produced by macrophages that infiltrate the adipose tissue, and its levels correlate to the degree of adiposity and insulin resistance. Deletion of TNF- $\alpha$ or TNF- $\alpha$ receptors (TNF- $\alpha-$ Rs) results in significantly improved insulin sensitivity suggesting a keyrole of TNF- $\alpha$ in obesity-related insulin resistance ${ }^{\mathbf{1 9}}$.

Peter et al., ${ }^{18}$ investigating relationships between changes in regional adiposity following lifestyle modification and circulating levels of SHBG among subjects at high-risk of T2DM, change in liver fat (quantified by magnetic resonance tomography) showed stronger correlations with serum SHBG than total body or visceral fat mass. Selvaet al., ${ }^{23}$ concluded that circulating levels of SHBG are determined by liver fat and not total body fat. 
In a previous study by Chubb et al. ${ }^{28}$ a negative correlation was seen between total cholesterol, triglyceride levels and SHBG.In this study, we could not find significant correlation. This is in agreement with Sá EQand his colleague $^{29}$ who found no significant correlation between SHBG and serum triglyceride levels or between SHBG levels and HDL. SHBG is possibly related to dyslipidemia, by regulating hepatic lipoprotein lipase activity and reducing the release of fatty acids from adipocytes ${ }^{30}$.

\section{Conclusion:-}

A low SHBG concentration is associated with hyperglycemia independently of insulinemia in T2DM. SHBG showed significant decreased level in both male and female. Our finding supports the association of circulating SHBG with type 2 diabetes.

Declaration of interest:-

Authors declare that they have no conflict of interest.

\section{References:-}

1. Ozougwu J. C, ObimbaK. C, Belonwu, C. D et al (2013). The pathogenesis and pathophysiology of type 1 and type 2 diabetes mellitus. Journal of Physiology and Pathophysiology. 4(4): 46-57.

2. Dorajoo R, LiuJ, BoehmBO. (2015). Genetics of Type 2 Diabetes and Clinical Utility. Genes 6, 372-384.

3. Arafa N, El Din G (2010). The epidemiology of diabetes mellitus in Egypt: Results of a National Survey .The Egyptian Journal of Community Medicine. 28: 3.

4. Szymezak J, Martin M, Leroy N, et al (2009).Carbamylated hemoglobin remains a critical issue in HbA1c measurements. ClinChem Lab Med. 47(5):612-613.

5. Le TN, Nestler JE, Strauss JF et al (2012). Sex hormone-binding globulin and type 2 diabetes mellitus.TrendsEndocrinolMetab. 23(1):32-40.

6. Yaghootkar H, Frayling TM(2013). Recent progress in the use of genetics to understand links between type 2 diabetes and related metabolic traits. Genome Biol. 28; 14(3):203.

7. Rosner W, Hryb DJ, Kahn SM, Nakhla AM, Romas NA(2010). Interactions of sex hormone-binding globulin with target cells.Mol Cell Endocrinol. 5; 316(1):79-85.

8. Chen B H, Brennan K, Goto A et al (2012). Sex Hormone-Binding Globulin and Risk of Clinical Diabetes in American Black, Hispanic, and Asian/Pacific Islander Postmenopausal Women.Clinical Chemistry. 58: 14571466.

9. 9. van GemertWA,Schuit AJ, van der Palen Jet al (2015). Effect of weight loss, with or without exercise, on body composition and sex hormones in postmenopausal women: the SHAPE-2 trial. Breast Cancer Res. 2; 17:120.

10. 10. Genuth S, Alberti KG, Bennett $\mathbf{P}$ (2003): Expert Committee on the Diagnosis and Classification of Diabetes Mellitus2, the Expert Committee on the Diagnosis and Classification of Diabetes Mellitus. Follow-up report on the diagnosis of diabetes mellitus. Diabetes Care. 26: 3160- 3167.

11. Perez-Fontan M, Cordido F, Rodriguez-Carmona $A$ et al (2004).Plasma ghrelin in patientsundergoninghaemodialysis and peritoneal dialysis. Nephrol Dial Transplant .4, 19(8): 2095-2100.

12. Mawi $M$ and Nirmalasari $\mathbf{R A ( 2 0 1 2 )}$ High free testosterone index increases lung function in adult males.UniversaMedicina. 31(2): 113-119.

13. Matthews DR, Hosker JP, Rudenski et al (1985). Homeostasis model assessment: insulin resistance and $\beta$ cell function from fasting plasma glucose and insulin concentrations in man. Diabetologia. 28:412-419.

14. Wallace IR, McKinley MC, Bell PM et al (2013).Sex hormone binding globulin and insulin resistance. Endocrinol (Oxf). 78(3):321-9.

15. Shin JY, Kim SK, Lee MY et al (2011). Serum sex hormone-binding globulin levels are independently associated with nonalcoholic fatty liver disease in people with type 2 diabetes. Diabetes Res ClinPract. 94:156162.

16. Saad F, Aversa A, Isidori A M et al. (2012). Testosterone as Potential Effective Therapy in Treatment of Obesity in Men with Testosterone Deficiency: A Review.Curr Diabetes Rev. 8(2): 131-143.

17. Ding EL, Song Y, Manson JEet al (2009): Sex hormone-binding globulin and risk of type 2 diabetes in women and men. New England Journal of Medicine. 361:1152-1163.

18. Peter A, Kantartzis K, MachannJ et al (2010). Relationships of circulating sex hormone-binding globulin with metabolic traits in humans.Diabetes. 59:3167-3173. 
19. Colangelo LA, Ouyang P, Liu Ket al(2009). Association of endogenous sex hormones with diabetes and impaired fasting glucose in men: multi-ethnic study of atherosclerosis. Diabetes Care.32 (6):1049-51.

20. Simó R, Barbosa-Desongles A, Lecube A, et al (2012).Potential Role of Tumor Necrosis Factor- $\alpha$ in Down regulating Sex Hormone-Binding Globulin.Diabetes. 61(2): 372-382.

21. 21.Chaoyang Li, Earl S. Ford, Benyi Li et al (2010).Association of Testosterone and Sex HormoneBinding Globulin With Metabolic Syndrome and Insulin Resistance in Men. Diabetes Care. 33:7 1618-1624

22. 22.Sutton-Tyrrell K, Wildman RP, Matthews KA et al (2005).Sex-hormone-binding globulin and the free androgen index are related to cardiovascular risk factors in multiethnic premenopausal and perimenopausal women enrolled in the Study of Women Across the Nation (SWAN). Circulation .111:1242-1249.

23. 23.Selva DM,HogeveenK N, Innis SMet al (2007). Monosaccharide-induced lipogenesis regulates the human hepatic sex hormone-binding globulin gene. J Clin Invest. 117:3979-3987.

24. 24.Osuna JA, Gómez-Pérez R, Arata-Bellabarbaet al (2006). Relationship between BMI, total testosterone, sex hormone-binding-globulin, leptin, insulin and insulin resistance in obese men.Arch Androl. 52(5):355-61

25. 25.Onat A, Hergenç G, Karabulut A et al (2007). Serum sex hormone-binding globulin, a determinant of cardiometabolic disorders independent of abdominal obesity and insulin resistance in elderly men and women. Metabolism. 56(10):1356-62.

26. 26.Aydın B and Winters SJ (2016). Sex Hormone-Binding Globulin in Children and Adolescents.J Clin Res PediatrEndocrinol. . 8(1): 1-12.

27. 27.KupelianV, Chiu G R, Araujo, et al (2007). Association of sex hormones and C-reactive protein levels in men.ClinEndocrino. 72(4): 527-533.

28. 28.Chubb SA, Hyde Z, Almeida OP et al (2008). Lower sex hormone-binding globulin is more strongly associated with metabolic syndrome than lower total testosterone in older men: the Health in Men Study. Eur J Endocrinol 158 (6):785-92.

29. 29.Sá EQ, Sá FC, Oliveira KCet al (2014).Association between sex hormone-binding globulin (SHBG) and metabolic syndrome among men. Sao Paulo Med J. 132(2):111-5.

30. 30.Desmeules A, Couillard C, Tchernof Aet al (2003).Post-heparin lipolytic enzyme activities, sex hormones and sex hormone-binding globulin (SHBG) in men and women: the HERITAGE Family Study. Atherosclerosis. 171: 343-350. 